The author has used the silver foil in a number of cases of brain surgery, and while no claim for anything new is made, he wishes to urge its more frequent use for the purpose indicated, and with greater care in reference to the technical details above mentioned.

The following case will illustrate the application of the principle:

George P., aged 12. When three years old he fell out of a chair and injured his head. He was taken to a hospital, where he remained two weeks. After returning home he was all right for about two years, when he developed epilepsy. The attacks were of the jacksonian type, beginning in the left arm. In addition there was partial paresis of certain muscles of the left arm, and to a very limited extent of the left leg. He entered my service at the Children's Hospital, June 22, 1900. Dr. H. T. Patrick saw the case with me, diagnosed a cortical lesion involving the left arm center, and advised operation. This was done July $10,1900$.

A large osteoplastic flap was turned down, uncovering the area indicated. A subdural calcareous mass was found about $3 \mathrm{cms}$. in diameter by $1 \frac{1}{2} \mathrm{cms}$. in thickness. It was firmly attached to the inner surface of the dura as well as to the brain tissue. A dural flap was turned down and freed from the mass. In removing the mass the cortical surface of the brain was unavoidably injured, and quite a depression remained in the brain tissue. After controlling the hemorrhage, the dura was replaced and sutured, as was also the osteoplastic tlap. Primary union occurred and recovery was without incident. He left the hospital Aug. 5, 1900.

Notwithstanding the fact that active treatment with the bromides was pushed after the operation, relief from the attacks only lasted about two months. They then recurred and gradually increased in frequency and severity until in February, 1902; when he again entered the hospital he was having from five to eight fits a day. The left arm was quite weak and movements impaired.

The second operation was performed February 17, 1902. The same osteoplastic flap was turned down, and the dura opened. Firm adhesions were found between the dura and the brain tissue. These were earefully separated and hemorrhage controlled. Several layers of very thin silver foil were then placed over the brain tissue, extending some distance beyond the area involved in adhesions. The dura was then replaced and sutured, and the skull closed as before. Recovery from the operation was perfect, and he left the hospital March 23, 1902. The bromid treatment was continued as before. He has been under observation up to the present time, and although it has been a year and ten months since the last operation, he has not only not had a single fit, but has improved materially in his left arm as well as in his general condition. He attends school regularly and says that he feels perfectly well.

\section{A METHOD OF DISPENSING WITH RUBBER GLOVES AND THE ADHESIVE RUBBER DAM.}

A PRELIMINARY NOTJE.

JOHN B. MURPHY, A.M., M.D.

Professor of Surgery Northwestern University Medical School Attending Surgeon Mercy Hospital, Cook County Hospital. CHICAGO.

The disadvantages, inconveniences and dangers of rubber gloves and dam, as well as their theoretical advantages, are well understood and will not be confmented on here. For several months past I have been endeavoring to find a material that might be applied to the hands of the surgeon and skin of the patient which would practically seal these surfaces with an insoluble, impervious and practically imperceptible coating-a coating that would not allow the secretions of the skin to escape and will not admit secretions, blood or pus into the pores or crevices of the skin, at the same time one that will not interfere with the sense of touch or impair the pliability of the skin. I have ascertained that a 4,6 or 8 per cent. solution of gutta-percha in benzine fulfills all of these requirements, while a similar solution in acetone also meets most of the requirements. In my clinical experience in the last four months I have found that the 4 per cent. solution of rubber in benzine is the most serviceable for the hands, as it wears better on the tips of the fingers under handling instruments, sponges and tissues than the acetone solution. For the abdomen the acetone solution has the advantage, as it dries in from three to four seconds after its application, while the benzine solution takes from two to three and a half minutes to dry to a firm coating. The acetone coating when dry is only slightly adhesive while the benzine coating is sticky.

The method of application to the hands and forearms is that of simple washing as with alcohol, care being taken to fill in around and beneath the nails. The hands must then be kept exposed to the air with the fingers separated until thoroughly dry. They may then be washed in alcohol, bichlorid or any of the antiseptic solutions without interfering with the coating or affecting the skin. It wears off on the tips of the fingers if the operations be many or prolonged, when another application may be made between operations; on the remaining portion of the hands one application is sufficient for a whole morning's work.

My routine method of hand preparation is as follows: First, five to seven minutes' washing with spirits of green soap (5 per cent.) and running hot water; second, three minutes' washing with alcohol; third, after thoroughly drying I pour on the rubber solution after the method above stated, allowing it to dry without rubbing, after the surface is thoroughly covered. The coating is so thin it can only be recognized by its glazed appearance. The coating will resist soap and water washing to cleanse the hands between operations. It is removed by washing in benzine.

The abdomen or surface preparation is five minutes' scrubbing with spirits of green soap (5 per cent.), then washing with ether, followed by alcohol. The surface is then swabbed over thoroughly with the acetone or benzine rubber solution.

The gutta-percha solution is prepared by dissolving pure gutta-percha chips in sterile benzine or acetone. This was accomplished first for me by the chemist, $\mathrm{E}$. von Hermann. These solutions do not stand boiling as it impairs the adhesiveness and elasticity of the coating. The advantages of this method of avoiding the dangers of infection from the hands and skin of the patient are very evident. In addition to the bactericidal properties of the benzine it prevents perspiration beneath the coated surface, and also the rubbing off of epithelia from the hands and skin surface into the wound. It does not puncture like the rubber glove, and where it wears off on the finger tips there is no accumulated epithelium or secretion beneath. It is, moreover, impermeable and precludes the ingress of infective flora or blood to the operator's skin. After operating, the surface washes clean as readily as the surface of a rubber glove. At the end of the day's work, when the hands are washed in benzine to remove the coating, the skin is very soft and smooth. The surgeon's hands are thus protected from all of the deleterious effects of daily operating, which is in itself a safeguard against infection. Inoculation and bacteriologic tests are being made and will be reported in detail 
later. The simplicity and ease of application of this method, with its practical certainty of protection, should appeal to every operator.

\section{SIMULTANEOUS EXTRA-U'TERINE AND IN- TRA-UTERINE PREGNANCY. \\ REPOIT OF A CASE. \\ CHARILS A. STILLWAGEN, M.D. \\ Gynecologist to East lind Hospital. \\ PITTSBULG, PA.}

Mrs. O., aged 38, was married Oet. 2, 1902, and had menstruated regularly every 24 days until April 20 , 1903, which was the date of her last period. She suffered more or less pelvic pain from that time. She was removed to St. Franeis Hospital July 14, 1903.

The following history was furnished me by that institution: Fanily history, negative. Personal history: She had always been delicate, and particularly so since an attack of pneumonia two years ago. Menstrual history was normal, the last period having occurred April 20, 1903. Present illness began about June 14 with obstinate constipation, and severe pain in abdomen centered over sigmoid region. Her physician gave morphia to relieve the pain. Later she had severe vomiting and was given fractional doses of ealomel without eflect. High enemas were administered with some effect. June 20, 1903, patient aborted two-months' fetus. The abdominal pain persisted, and was most marked in the lower right quadrant; abdominal muscles were rigid. She was admitted to the hospital July 14, suffering from almost continuous vomiting, constipation amounting to obstruction and severe abdominal and lumbar pain. She was thin and emaciated, and of a sallow complexion.

Examination of the heart was negative. There was impaired resonance at the apex of the right lung in front and over the left apex posteriorly; bronchovesicular breathing posteriorly on the left side, anteriorly on the riglit side and suberepitant râles on the right side anteriorly. The abdomen was distended, tender on pressure, rigid, dull in the flanks and over the hypogastrium. No tumefaction could be outlined. An evacuation of the bowels was secured July 16, after which all symptoms were somewhat relieved, but the patient becane dissatisfied and returned to her home July 20.

At her home a diagnosis of abdominal pregnancy was made by Dr. R. M. Sands, who sent her to the East End Hospital, for operation, where she was admitted August 6. Throughout her illness morphia had been administered only in sufficient quantities to relieve pain, but she had become more or less addicted to the drug. She was emaciated to the last degree. A large hard mass could be felt in the right iliac region which protruded so that it could be distinctly palpated. No fetal heart sounds or bruit could be heard. The appearance of the mass and the patient's cachexia suggested malignant disease.

An operation was performed August 8 . The incision was made in the median line. Firm intestinal adhesions were dissected from the anterior surface of the mass before the enveloping membranes were revealed. Haste being such an important factor on account of the desperate physical condition of the patient, these were simply incised, the liquor amnii evacuated, and a perfectly fresh fetus with the cord and placenta removed. The sac was packed with iodoform gauze to control hemorrhage, and the edges sutured in the abdominal wound. The fetus measured $10.5 \mathrm{~cm}$. from vertex to rump, and was probably alive when delivered.

The patient was almost pulseless when taken from the operating table, but reacted nicely after free stimulation and hypodermoclysis. The temperature and pulse rapidly reached normal; bowels moved on the fourth day. The gauze was removed on the fourth day and fecal matter was found in it. The membranes sloughed away on the eighth day, the fecal fistula persisting. Convalescence was further complicated by the morphia habit previously mentioned. The patient was exceedingly hard to manage and insisted on returning to her home, which she was permitted to do on her promise to have the care of a trained nurse. At this time, thirty-nine days after operation, she was able to be about her room and the halls of the hospital. After her return home she declined the attention of a nurse, finally refused nourishment entirely, and died of inanition, Oct. 19, 1903, sixty-seven days after operation.

A thorough autopsy was not practicable, but a sufficient examination was made by Dr. James Ralston to determine the primary seat of the abdominal pregnancy as right tuboovarian. The fistulous tract communicated with the rectum and was probably due to a placental attachment at that point.

The condition which this case illustrated is the simultancous development of an intra-uterine and an extrauterne gestation. That fecundation is also practically simultancous in the great majority of cases is borne out by the reports of unimpeachable cases to date. It is the phenomenon of a twin pregnancy, except that the one ovum is arrested in its course before it reaches the uterine cavity. No pathologic condition can be especially charged with its occurrence, except those which obtain in the ordinary cases of ectopic pregnancy. Cases of intra-uterine pregnancy occurring after the termination of an extra-uterine gestation do not come under this classification.

Simultaneous extra-uterine and intra-uterine pregnancy is looked on as a rare condition, and some of the latest text-books on gynecology dismiss the subject with a line and speak of it merely as one of the possibilities. Zinke ${ }^{1}$ tabulated 88 cases taken from the collections of B. B. Browne, H. Gutzwiller, John S. Parry, E. W. Martin and Christer-Nilson, to which he adds his own collection with the report of 1 case. Vilsin ${ }^{2}$ collected from the literature as far back as the eleventh century 68 cases, to which he adds 1 observation of his own. In 58 per cent. of the cases of this collection the women were between 30 and 40 years of age; 19 per cent. were primiparæ. In only 20 cases did both fetuses arrive at full term; in 47 both aborted, usually one before the other. The death of the mother caused the death of the immature fetus 6 times, i. e., in 10 per cent. of the cases; the intra-uterine fetus went to term in 29 cases, i. e., in 46 per cent. of the cases. In 25 cases, or $3 \%$ per cent., the extra-uterine embryo went to full term. Vilsin's most important conclusions are that interruption of the intra-uterine pregnancy had by far a slighter effect on the further development of the extra-uterine pregnancy than vice versa.

Disturbances in the blood circulation in close neighborhood to the uterus caused by rupture or abortion of the pregnant tube, are in many cases responsible for the premature interruption of the intra-uterine pregnancy. The fact that in twenty-five cases the ectopic fetus reached a full term is not considered by Engstrom ${ }^{3}$ as a mere accident, but he believes that increased afflux of blood to the pelvis on account of the intra-uterine pregnancy improves at the same time the blood supply of the pregnant tube, thus promoting the development of the ectopic fetus.

At least twenty-five cases of simultaneous extrauterine and intra-uterine pregnancy may be added to the tabulated record of Zinke, mentioned above. Some of these were overlooked in the preparation of the various collections and additional ones have been reported since the date of his collection on going to print. But it is more than probable that the reported cases of this

1. Journal of Obstetrics, May, 1902.

2. Ibid., January, 1902.

3. Amer. Year Book of Med. and Surg., 1903 